\title{
Evaluación de la eficacia de fosfomicina trometamol y gentamicina en la profilaxis de la cistoscopia flexible tras el estudio de los patógenos de nuestro entorno
}

\section{Evaluation of the Efficacy of Fosfomycin Trometamol and Gentamicin in the Prophylaxis of Flexible Cystoscopy after the Study of Microflora in our Environment}

Jorge Planelles Gómez ${ }^{1}$ Elena Gras Colomer ${ }^{2}$ María Ángeles Pérez Martínez ${ }^{1}$ Cristina Bonastre Torró ${ }^{1}$ Manuel Sánchez Sanchís ${ }^{1}$ Juan Francisco Vidal Moreno ${ }^{1}$

\footnotetext{
${ }^{1}$ Médico Adjunto del Servicio de Urología del Hospital Universitario Doctor Peset de Valencia, Valencia, España

2 Farmacéutica del Servicio de Farmacia del Hospital Universitario Doctor Peset de Valencia, Valencia, España
}

Address for correspondence Jorge Planelles Gómez, Médico Adjunto (MD), Servicio de Urología del Hospital Universitario Doctor Peset de Valencia, Gran vía Ramón y Cajal 5, 21, Valencia 46007, España (e-mail: jorge_planelles@yahoo.es).

Urol Colomb 2019;28:260-268.

\begin{abstract}
Resumen
Palabras Clave

- profilaxis

- cistoscopia

- fosfomicina trometamol

- gentamicina

- infección del tracto urinario

- antibiótico

Objetivo Optimizar el uso de antibióticos en la profilaxis de la cistoscopia flexible estudiando los patógenos más frecuentes de nuestro entorno y eligiendo el antibiótico según sus antibiogramas.

Métodos Desde Enero del 2015 hasta Noviembre del 2015, se analizaron los urinocultivos de nuestra área, se eligió el antibiótico en función a su sensibilidad frente a los patógenos más frecuentes y se comparó con un antibiótico de amplio espectro. Desde Enero del 2016 hasta Diciembre del 2016, se realizaron las cistoscopias agrupando a los pacientes en: Grupo 1: Pacientes sin profilaxis; Grupo 2: Profilaxis con Gentamicina 240 mg; Grupo 3: Profilaxis con antibiótico seleccionado. Como variables principales se definieron la presencia de bacteriuria asintomática e ITU tras la realización de la cistoscopia flexible.

Resultados Se analizaron 8.530 urinocultivos y se eligió la Fosfomicina Trometamol 3 gr como profilaxis. Se realizaron 244 cistoscopias distribuidas: Grupo 1: 86 (35\%); Grupo 2: 72 (30\%); Grupo 3: 86 (35\%). Se detectó bacteriuria asintomática postcistoscopia en 6 pacientes (2,5\%) en el Grupo 1, 7 pacientes (2,9\%) en el grupo 2 y 5 pacientes (2\%) en el grupo 3 no presentando diferencias significativas ( $p 0.120)$. Desarrollaron ITUs postcistoscopia 1 paciente $(0,4 \%)$ en el Grupo 1,5 pacientes $(2 \%)$ en el Grupo 2 y 2 pacientes ( $0,8 \%$ ) en el Grupo 3 sin diferencias significativas ( $p$ 0.105). Conclusión La Fosfomicina es tan efectiva como la Gentamicina en la profilaxis de la cistoscopia. Para un uso correcto de los antibióticos, se recomienda el estudio de los patógenos de nuestro entorno.
\end{abstract}

received

June 21, 2017

accepted

September 18, 2017
DOI https://doi.org/ $10.1055 / \mathrm{s}-0038-1651508$. ISSN 0120-789X. eISSN 2027-0119.
Copyright (c) 2019, Sociedad Colombiana License terms de Urología. Publicado por Thieme Revinter Publicações Ltda., Rio de Janeiro, Brazil. Todos los derechos reservados. 
Abstract

\section{Keywords}

- prophylaxis

- cystoscopy

- fosfomycin trometamol

- gentamicin

- urinary tract infection

- antibiotic
Objective To optimize the use of antibiotics in the prophylaxis of flexible cystoscopy by studying the most frequent pathogens in our environment and choosing the antibiotic according to its antibiograms.

Method Between January 2015 and November 2015, urine cultures were analyzed in our area, the antibiotic was chosen based on its sensitivity to the most frequent pathogens and compared with a broad spectrum antibiotic. From January 2016 to December 2016, cystoscopy was performed by grouping patients into: Group 1 Patients without prophylaxis, Group 2 - Prophylaxis with 240 mg gentamicin, Group 3 Selected antibiotic prophylaxis. The main variables were the presence of asymptomatic bacteriuria and UTI after flexible cystoscopy.

Results 8530 urine cultures were analyzed and $3 \mathrm{~g}$ of fosfomycin trometamol was chosen as the prophylactic. There were 244 cystoscopies: Group 1: 86 (35\%); Group 2: 72 (30\%); Group 3: 86 (35\%). Asymptomatic bacteriuria was detected in 6 patients (2.5\%) in Group 1, 7 patients (2.9\%) in Group 2 and 5 patients (2\%) in Group 3, showing no significant differences $(p=0.120)$. Post-cystoscopic urinary tract infection developed in 1 patient $(0.4 \%)$ in Group 1,5 patients (2\%) in Group 2 and 2 patients $(0.8 \%)$ in Group 3, which showed no significant differences ( $p$ 0.105).

Conclusion Fosfomycin is as effective as Gentamicin as a prophylactic in cystoscopy. The study of the pathogens in each environment is recommended to correctly prescribe the antibiotic.

\section{Introducción}

La cistoscopia flexible es una prueba fundamental y rutinaria en la monitorización del tumor vesical superficial así como en el diagnóstico de patologías como la estenosis uretral, hematuria o los síntomas del tracto urinario inferior. Sin embargo, la cistoscopia es una prueba invasiva, clasificada como limpiacontaminada ${ }^{1}$ por lo que se recomiendan medidas de asepsia estándar.

La literatura es muy variable con respecto a la incidencia de la Infección del Tracto Urinario (ITU) tras la realización de una cistoscopia rondando entre el $2 \%$ y el $15 \%$, ${ }^{2,3}$ pero hay autores que registran una incidencia superior al 30\%. ${ }^{4}$

Existe controversia en cuanto a la necesidad de la profilaxis en la cistoscopia flexible para reducir las bacteriurias asintomáticas o infecciones menores levemente asintomáticas que se resuelven espontáneamente en pacientes sin factores de riesgo. $^{4-7}$

Tampoco existe un criterio unánime en cuanto al tipo, pauta y administración de los antibióticos debido en parte a las diferentes microfloras de cada entorno. ${ }^{8}$ Además, el uso indiscriminado de antibióticos en los últimos años ha producido que microorganismos sensibles hayan mutado creando resistencias a antibióticos que clásicamente se utilizaban para la profilaxis como las Fluoroquinolonas o el Trimetropim Sulfametoxazol (TMP + SMX), sobre todo en el sur de Europa. ${ }^{6,9}$ En ese sentido, es necesario un uso racional de los antibióticos en la quimioprofilaxis ajustado a las recomendaciones que se hacen en la guías de práctica clínica, y en ellas, no se recomienda la quimioprofilaxis de rutina previa a la cistoscopia flexible excepto en aquellos pacientes con factores de riesgo asociados. ${ }^{1}$

La Gentamicina es un aminoglucósico de amplio espectro activo frente a los uropatógenos más frecuentes ya utilizados anteriormente como quimioprofilaxis en intervenciones urológicas, pero con el inconveniente de tener que ser administrado vía parenteral o intramuscular, además de ser ototóxico y nefrotóxico. ${ }^{10}$

El objetivo principal de nuestro estudio es la selección de un antibiótico para la profilaxis de la cistoscopia flexible, basado en el estudio de la microflora de nuestro entorno, y compararlo con una quimioprofilaxis estándar de amplio espectro como la Gentamicina $240 \mathrm{mg}$ intramuscular.

\section{Material y Métodos}

Se trata de un estudio unicéntrico prospectivo, semiexperimental entre Enero del 2015 y Diciembre del 2016. El estudio se realizó previa obtención del consentimiento informado de la prueba por parte de los pacientes.

\section{Estudio de la Microflora}

La elección del antibiótico para la quimioprofilaxis se basó en el análisis descriptivo desde Enero del 2015 hasta noviembre del 2015 de los patógenos más frecuentes de nuestra área. Se incluyeron los urinocultivos y sus respectivos antibiogramas procedentes de los pacientes que acudieron a los servicios de atención primaria de nuestra zona con la sospecha de ITU durante ese periodo, y los urinocultivos solicitados durante el periodo de febrero 2015 hasta mayo 2015 de los pacientes 
que acudieron a nuestro servicio a realizarse una cistoscopia flexible.

\section{Tratamiento Profiláctico en la Cistoscopia Flexible}

Se eligió aquel antibiótico que fuera activo frente a los microorganismos más frecuentes que se pudiera administrar vía oral y que tuviera bajo perfil de toxicidad o de reacciones adversas.

Los pacientes sin factores de riesgo no recibieron quimioprofilaxis durante el periodo de estudio, tal y como establecen las guías de práctica clínica. Como factores de riesgo se consideraron: mayores de 75 años, inmunosupresión, diabéticos, portadores de cualquier tipo de catéteres, antecedentes de de ITU o litiasis y sospecha de obstrucción con residuos altos.

Los pacientes que cumplían alguno de esos factores fueron tratados con 2 antibióticos según dos periodos de estudio:

- La Fase I se desarrolló entre enero del 2016 hasta abril del 2016. A los pacientes con factores de riesgo a los que se les realizó una cistoscopia flexible, se les administró quimioprofilaxis con Gentamicina $240 \mathrm{mg}$ intramuscular, tal y como se realizaba en la práctica habitual de nuestro servicio.

- La Fase II se desarrolló inicialmente entre julio del 2016 hasta octubre del 2016, precisando prolongar el periodo hasta diciembre del 2016 para obtener grupos con $n$ similar. A esos pacientes se les administró el antibiótico elegido tras el estudio de la microflora.

\section{Cistoscopia}

Las cistoscopias se realizaron mediante cistoscopio flexible de 17 ch (Sumedex) en condiciones de asepsia, tras el lavado de los genitales con clorhexidina.

A su llegada a la consulta, se comprueba la identidad del paciente y se recogen las siguientes variables de cada uno de ellos: factores de riesgo; posibles alergias a antibióticos; presencia de síntomas urinarios previos de llenado, de vaciado, hematuria o fiebre; tabaquismo; motivo de la cistoscopia; posibles tratamientos intravesicales; manipulaciones urológicas 3 meses antes y número de cistoscopias previas.

A los pacientes con factores de riesgo se les administró el antibiótico elegido o la Gentamicina $240 \mathrm{mg}$ vía intramuscular 15-30 minutos antes de la prueba.

Tras la introducción del cistoscopio, se extrajo la muestra para el urinocultivo, se realizó la cistoscopia, se tomó nota del resultado de la misma y se programó al paciente para el siguiente control.

Se le dio un urinocultivo a realizar 10 días después.

Quince días después de la realización del procedimiento, se les entrevistó telefónicamente y se les preguntó si habían presentado síntomas del tracto urinario tras la cistoscopia, duración de los síntomas y si habían precisado consultar a algún profesional sanitario necesitado ingreso hospitalario.

\section{Variables del Estudio}

El cálculo del tamaño muestral se calculó mediante la proporción esperada de ITU del tratamiento estándar $11 \%$ con un alfa de 0,05 y $\beta$ del 0,20 y con una proporción esperada de la profilaxis del antibiótico apropiado para la flora de nuestro medio del $1 \%$ (disminución del $10 \%$ ), requiriendo un tamaño muestral mínimo en cada grupo de 69 pacientes.

Como variables principales, se definieron la presencia de bacteriuria asintomática o ITU tras la realización de la cistoscopia flexible. Se define como bacteriuria significativa precistoscopia la presencia de $10 \times 2$ UFC 0 más de la orina extraída directamente del cistoscopio. Como bacteriuria significativa postcistoscopia la presencia de $10 \times 5$ UFC o más en la muestra de orina por micción espontánea realizada a los 10 días. Se define como Infección del Tracto Urinario pre o postcistoscopia la presencia de bacteriuria significativa más síntomas del tracto urinario, y como bacteriuria asintomática aquella que presenta bacteriuria significativa sin la presencia de síntomas.

Para estudiar la aparición de nuevas ITUs, se eliminaron aquellas ITUs postcistoscopias que presentaron criterios de ITU precistoscopias al considerar que el patógeno ya estaba presente antes de la misma.

\section{Análisis Estadístico}

El análisis estadístico se realizó mediante el software estadístico SPSS v.19. (Chicago Inc.) Se analizó la normalidad de las variables mediante la prueba de kolmorogov-smirnov. Las variables con distribución normal se mostraron como media y desviación estándar (sd), y las no normales como mediana y rango intercuartil(IQR). Las variables cualitativas se presentaron en porcentaje.

El análisis principal comparó la bacteriuria después de la cistoscopia en los tres grupos de tratamiento; las diferencias entre los grupos se evaluaron utilizando la prueba de chicuadrado para variables cualitativas y el test de t student de muestras independientes para variables cuantitativas. Se calculó el riesgo relativo de padecer ITU entre los tratamientos a través de regresión logística. Se calculó el número de pacientes que es necesario tratar (NNT), definido como el número de pacientes que se estima que es necesario tratar con el nuevo tratamiento, en lugar de con el tratamiento con Gentamicina 240 mg. Para prevenir un suceso, se calculó como la inversa de la reducción absoluta del riesgo.

\section{Resultados}

\section{Estudios de la Microflora}

En el estudio previo de nuestra microflora se analizaron un total de 8.530 urinocultivos. Los patógenos más frecuentes fueron: Escherichia coli (EC) 29,93\%, Enterococcus faecalis $13,13 \%$ y Klebsiella pneumoniae $7,87 \%$. Sus respectivos antibiogramas se encuentran resumidos en la -Tabla 1.

Tras el análisis del estudio descriptivo de nuestros patógenos, se eligió la Fosfomicina Trometamol (FT) de $3 \mathrm{~g}$ como antibiótico para la profilaxis.

\section{Tratamiento Profiláctico en la Cistoscopia Flexible}

Se incluyeron 244 pacientes ( 40 mujeres y 204 hombres), con una edad media de 68,41 años durante el periodo entre enero de 2016 y diciembre de 2016. Ochenta y seis (86) pacientes 
Tabla 1 Patógenos más frecuentes y sensibilidad antibiótica

\begin{tabular}{|c|c|c|c|c|c|c|c|}
\hline Total de aislamientos & \multicolumn{3}{|l|}{8.530} & & & & \\
\hline Especie & \multicolumn{3}{|c|}{$\mathrm{N}^{\circ}$ cepas } & \multicolumn{2}{|l|}{$\%$} & \multicolumn{2}{|c|}{$\%$ acumulado } \\
\hline Escherichia coli & \multicolumn{3}{|l|}{2.553} & \multicolumn{2}{|l|}{$29,93 \%$} & \multicolumn{2}{|l|}{$29,93 \%$} \\
\hline Enterococcus faecalis & \multicolumn{3}{|l|}{1.120} & \multicolumn{2}{|l|}{$13,13 \%$} & \multicolumn{2}{|l|}{$43,06 \%$} \\
\hline Klebsiella pneumoniae & \multicolumn{3}{|l|}{671} & \multicolumn{2}{|l|}{$7,87 \%$} & \multicolumn{2}{|l|}{$50,93 \%$} \\
\hline Gardnerella vaginalis & \multicolumn{3}{|l|}{632} & \multicolumn{2}{|l|}{$7,41 \%$} & \multicolumn{2}{|l|}{$58,34 \%$} \\
\hline Candida albicans & \multicolumn{3}{|l|}{626} & \multicolumn{2}{|l|}{$7,34 \%$} & \multicolumn{2}{|l|}{$65,67 \%$} \\
\hline Streptococcus agalactiae B & \multicolumn{3}{|l|}{425} & \multicolumn{2}{|l|}{$4,98 \%$} & \multicolumn{2}{|l|}{$70,66 \%$} \\
\hline Proteus mirabilis & \multicolumn{3}{|l|}{321} & \multicolumn{2}{|l|}{$3,76 \%$} & $74,42 \%$ & \\
\hline Pseudomonas aeruginosa & 280 & & & $3,28 \%$ & & $77,70 \%$ & \\
\hline Escherichia coli BLEE & 235 & & & $2,75 \%$ & & $80,46 \%$ & \\
\hline Streptococcus pyogenes A & 208 & & & $2,44 \%$ & & $82,90 \%$ & \\
\hline Staphylococcus aureus & 205 & & & $2,40 \%$ & & $85,30 \%$ & \\
\hline Ureaplasma urealyticum & 131 & & & $1,54 \%$ & & $86,83 \%$ & \\
\hline Enterobacter cloacae & 109 & & & $1,28 \%$ & & $88,11 \%$ & \\
\hline Morganella morganii & 98 & & & $1,15 \%$ & & $89,26 \%$ & \\
\hline Klebsiella oxytoca & 87 & & & $1,02 \%$ & & $90,28 \%$ & \\
\hline Escherichia coli & & & & Enterococcus faecalis & & & \\
\hline & 2.553 & & & & 1.120 & & \\
\hline Antimicrobianos & Total & $\% \mathrm{~S}$ & $\% \mathrm{R}$ & Antimicrobianos & Total & $\% \mathrm{~S}$ & $\% \mathrm{R}$ \\
\hline A. Nalidíxico & 259 & $52,51 \%$ & $47,49 \%$ & Ampicilina & 1.111 & $100,00 \%$ & $0,00 \%$ \\
\hline Amikacina & 2.433 & $98,89 \%$ & $1,11 \%$ & Cefuroxima & 163 & $0,00 \%$ & $100,00 \%$ \\
\hline Amoxi/Clav. & 2.549 & $80,23 \%$ & $7,30 \%$ & Ciprofloxacina & 1.110 & $70,90 \%$ & $26,58 \%$ \\
\hline Ampicilina & 2.549 & $40,96 \%$ & $58,45 \%$ & Clindamicina & 167 & $1,20 \%$ & $98,80 \%$ \\
\hline Cefalotina & 119 & $36,13 \%$ & $28,57 \%$ & Cloranfenicol & 170 & $89,41 \%$ & $9,41 \%$ \\
\hline Cefepime & 255 & $90,98 \%$ & $9,02 \%$ & Cotrimoxazol & 166 & $0,60 \%$ & $99,40 \%$ \\
\hline Cefotaxima & 2.551 & $97,33 \%$ & $2,04 \%$ & Daptomicina & 929 & $99,89 \%$ & $0,00 \%$ \\
\hline Cefoxitina & 2.545 & $92,93 \%$ & $2,91 \%$ & Eritromicina & 168 & $11,90 \%$ & $47,02 \%$ \\
\hline Ceftazidima & 2.551 & $97,49 \%$ & $2,12 \%$ & Fosfomicina & 897 & $100,00 \%$ & $0,00 \%$ \\
\hline Cefuroxima & 2.548 & $89,64 \%$ & $5,61 \%$ & Imipenem & 175 & $100,00 \%$ & $0,00 \%$ \\
\hline Ciprofloxacina & 2.551 & $69,23 \%$ & $29,71 \%$ & Levofloxacina & 1.111 & $73,09 \%$ & $26,01 \%$ \\
\hline Cotrimoxazol & 2.551 & $72,29 \%$ & $27,71 \%$ & Linezolid & 1.102 & $99,27 \%$ & $0,09 \%$ \\
\hline Ertapenem & 2.546 & $99,76 \%$ & $0,16 \%$ & Nitrofurantoina & 1.108 & $97,47 \%$ & $0,90 \%$ \\
\hline Fosfomicina & 2.425 & $95,18 \%$ & $4,82 \%$ & Penicilina & 1.112 & $99,82 \%$ & $0,18 \%$ \\
\hline Gentamicina & 2.550 & $89,18 \%$ & $9,84 \%$ & Quinu/Dalfopristina & 167 & $0,00 \%$ & $100,00 \%$ \\
\hline Imipenem & 343 & $100,00 \%$ & $0,00 \%$ & Sinergismo Gentamicina & 1.108 & $70,67 \%$ & $29,33 \%$ \\
\hline Levofloxacina & 2.301 & $70,88 \%$ & $25,12 \%$ & Sinergismo Streptomicina & 1.108 & $72,29 \%$ & $27,71 \%$ \\
\hline Meropenem & 2.300 & $99,96 \%$ & $0,00 \%$ & Teicoplanina & 1.111 & $100,00 \%$ & $0,00 \%$ \\
\hline Nitrofurantoina & 2.413 & $96,35 \%$ & $1,70 \%$ & Tetraciclina & 934 & $11,78 \%$ & $87,04 \%$ \\
\hline Norfloxacina & 2.296 & $64,68 \%$ & $35,32 \%$ & Tigeciclina & 168 & $100,00 \%$ & $0,00 \%$ \\
\hline Piper/Tazo. & 2.434 & $96,84 \%$ & $1,40 \%$ & Vancomicina & 1.111 & $99,91 \%$ & $0,00 \%$ \\
\hline Tigeciclina & 134 & $97,76 \%$ & $0,00 \%$ & & & & \\
\hline Tobramicina & 2.416 & $89,24 \%$ & $6,42 \%$ & & & & \\
\hline
\end{tabular}


(35\%), no presentaron factores de riego por lo que no se les trató con antibióticos, ya 158 presentaron factores de riesgos, de los cuales 72 (30\%) se trataron con Gentamicina $240 \mathrm{mg}$ durante el primer periodo y 86 (35\%) con FT 3 g durante el segundo periodo.

En la - Tabla 2 se recogen las características de los pacientes, en la que no se evidencian diferencias significativas entre los grupos de tratamiento que se comparan excepto en la presencia de HBP que mostró diferencias entre los grupos activos de tratamiento.

En cuanto a las variables principales, se detectó bacteriuria asintomática postcistoscopia en 6 (2,5\%) pacientes en el grupo sin profilaxis, $7(2,9 \%)$ en el grupo con profilaxis con Gentamicina y 5 (2\%) en el grupo de FT no presentando diferencias estadísticamente significativas con una p 0.120 .

Un total de 5 pacientes (2\%) en el grupo tratado con Gentamicina, 2 pacientes $(0,8 \%)$ en el grupo tratado con FT y 1 paciente $(0,4 \%)$ sin profilaxis, desarrollaron criterios de ITU tras las cistoscopias, no mostrando diferencias estadísticamente significativas (p 0.105). Además, si excluimos a los pacientes que ya presentaban criterios de ITU el día de la cistoscopia, solamente hubo 1 paciente con FT, 3 con Gentamicina y 1 del grupo sin profilaxis que mostraron verdaderas ITU de novo tras la realización de la cistoscopia flexible.
De esos 8 casos de ITU, 2 pacientes necesitaron asistencia por un profesional sanitario pero de forma ambulatoria. De los 244 pacientes del estudio, 45 (10,98\%) presentaron síntomas urinarios tras la realización de la cistoscopia por cualquier causa, en 8 de ellos tuvieron que consultar al médico, y en solamente en 10 de esos pacientes, los síntomas duraron más de 48 horas.

En los 8 casos de ITU postcistoscopia, los patógenos implicados fueron Escherichia coli en el 62,5\% (5 pacientes), Enterococcus faecalis en el 12,5\% (1 paciente) y Pseudomona aeruginosa en el $12,5 \%$ ( 1 paciente). 1 paciente (12,5\%) fue registrado como otros patógenos. En los 18 casos de bacteriuria asintomática hubo 7 casos $(38,88 \%)$ con cultivo positivo a Enterococcus faecalis, 5 casos (27,77\%) de Escherichia coli, 1 caso $(5,55 \%)$ de Klebsiella pneumoniae, 1 caso $(5,55 \%)$ de Proteus mirabillis y 4 casos $(22,22 \%)$ de otros patógenos.

El riesgo calculado de padecer ITU es $1,2 \%$ para la profilaxis con FT y 4\% para Gentamicina. En ese sentido, el NNT es 35, es decir, tratando a 35 pacientes con FT en lugar de con Gentamicina evitaremos una infección urinaria.

\section{Discusión}

La cistoscopia es probablemente el procedimiento diagnóstico más frecuente realizado en un servicio de

Tabla 2 Distribución de pacientes y resultados

\begin{tabular}{|c|c|c|c|c|c|}
\hline & & $\begin{array}{l}\text { G1 } \\
\text { Sin profilaxis }\end{array}$ & $\begin{array}{l}\text { G2 } \\
\text { Gentamicina } \\
240 \mathrm{mg}\end{array}$ & $\begin{array}{l}\text { G3 } \\
\text { Fosfomicina } \\
3 \mathrm{~g}\end{array}$ & $p(G 2$ vs G3) \\
\hline \multicolumn{2}{|l|}{ Pacientes n (\%) } & $86(35 \%)$ & $72(30 \%)$ & $86(35 \%)$ & - \\
\hline \multicolumn{2}{|c|}{ Edad (años) media (SD) } & $62,65(8,72 \%)$ & $68,77(11,21 \%)$ & $73,83(7,72 \%)$ & 0,123 \\
\hline \multirow[t]{2}{*}{ Sexo } & Masculino & $66(27,0 \%)$ & $61(25,0 \%)$ & $77(31,6 \%)$ & \multirow[t]{2}{*}{0,365} \\
\hline & Femenino & $20(8,2 \%)$ & $11(4,5 \%)$ & $9(3,7 \%)$ & \\
\hline \multicolumn{6}{|l|}{ Factores de riesgo } \\
\hline \multicolumn{2}{|l|}{ Edad $>75$} & - & $39(45 \%)$ & 47 (55\%) & 0,951 \\
\hline \multirow[t]{3}{*}{ Hábito tabáquico } & Sí & $31(13 \%)$ & $13(5 \%)$ & $15(6 \%)$ & \multirow[t]{3}{*}{0,998} \\
\hline & Exfumador & $36(15 \%)$ & $41(17 \%)$ & $50(20 \%)$ & \\
\hline & No & $19(8 \%)$ & $18(7 \%)$ & $21(9 \%)$ & \\
\hline \multicolumn{2}{|l|}{ Diabetes } & - & $23(9,43 \%)$ & $37(14,16 \%)$ & 0,153 \\
\hline \multicolumn{2}{|l|}{ ITU repetición } & - & $16(6,56 \%)$ & $21(8,61 \%)$ & 0,745 \\
\hline \multicolumn{2}{|l|}{ Inmunosupresión } & - & $4(1,64 \%)$ & $7(2,87 \%)$ & 0,525 \\
\hline \multicolumn{2}{|l|}{$\mathrm{HBP}$} & $12(4,92 \%)$ & $16(6,56 \%)$ & $39(8,61 \%)$ & $0,020^{*}$ \\
\hline \multicolumn{2}{|l|}{ Litiasis } & - & $7(2,87 \%)$ & $4(1,64 \%)$ & 0,212 \\
\hline \multicolumn{2}{|l|}{ Catéter } & - & $2(0,82 \%)$ & $2(0,82 \%)$ & 0,857 \\
\hline \multicolumn{2}{|l|}{ Menopausia } & $14(5,74 \%)$ & $11(4,51 \%)$ & $10(4,10 \%)$ & 0,501 \\
\hline \multicolumn{2}{|c|}{ Cirugía último trimestre } & $9(3,69 \%)$ & $16(6,56 \%)$ & $21(8,61 \%)$ & 0,745 \\
\hline \multicolumn{6}{|l|}{ Resultados } \\
\hline \multicolumn{2}{|c|}{ ITU postcistoscopia (\%) } & $1(0,4 \%)$ & $5(2 \%)$ & $2(0,8 \%)$ & 0,105 \\
\hline \multicolumn{2}{|c|}{$\begin{array}{l}\text { Bacteriuria asintomática } \\
\text { postcistoscopia (\%) }\end{array}$} & $6(2,5 \%)$ & 7 (2,9\%) & $5(2 \%)$ & 0,120 \\
\hline
\end{tabular}

*Diferencia significativa. 
urología. Al ser un procedimiento invasivo es lógico pensar que se requiera de una profilaxis antibiótica. No obstante, la mayoría de la literatura recoge una incidencia de ITU del $2 \%$ al $10 \%^{2-4,11}$ y una incidencia de bacteriurias significativas entre $8 \%-18 \%{ }^{4,12}$ La mayor parte de esos procesos, no son graves y no requieren ni siquiera un tratamiento activo. Por lo tanto el debate surge al determinar si es necesario reducir esa incidencia mediante profilaxis o si con las medidas de asepsia es suficiente. En nuestro estudio, la tasa de ITU con la administración de FT como quimoprofilaxis fue del 0,8\%, inferior a la registrada en la bibliografía, y se demuestra que esa tasa no muestra diferencias estadísticamente significativas que las conseguidas con la quimioprofilaxis con un antibiótico de amplio espectro como la Gentamicina $240 \mathrm{mg}$.

Parece que hay consenso en que no es necesaria la profilaxis con antibiótico de rutina, administrándola solo en aquellos pacientes que presenten factores de riesgo descritos por la American Society of Anestesiology (ASA). ${ }^{1}$ Donde no existe unanimidad es en cuanto al tipo de antibiótico, dosis o pauta de administración del mismo, en parte debido a las diferentes microfloras que hay en Europa. Las guías europeas recomiendan la profilaxis antes de una cistoscopia flexible mediante Trimetropim-Sulfametoxazol (TMP + SMX), Cefalosporinas de $2^{\mathrm{a}}$ generación 0 Nitrofurantoína. ${ }^{1}$ En nuestra serie, solamente 1 paciente $(0,4 \%)$ sin factores de riesgo y no tratados con profilaxis antibiótica desarrolló ITU postcistoscopia. Esa tasa no mostró diferencias estadísticamente significativas con respecto a los pacientes con quimioprofilaxis, lo que corrobora la no necesidad de administrar antibiótico en ese tipo de pacientes.

Existen diferencias muy importantes entre las distintas microfloras de los países de Europa. Además, en los países del Mediterráneo hay una gran resistencia bacteriana debido al uso indiscriminado de antibióticos. ${ }^{9}$

Tanto las guías europeas como los diversos estudios epidemiológicos europeos, describen estos uropatógenos como los más frecuentes implicados en una posible ITU tras una cistoscopia : Escherichia coli (EC), Klebsiella spp., Proteus, otras Enterobacteriaceae, S. saprophyticus y, menos frecuentemente las Enterococci. ${ }^{1,9}$ Esos patógenos han demostrado un aumento en la resistencia a antibióticos tradicionales como ampicilina y TMP + SMX, $, 13,14$ y en los últimos años a Quinolonas, en algunos países. ${ }^{15,16}$ Entre ellos España, que presenta las resistencias más altas a Quinolonas, sobre todo el EC y solamente es adelantada por Portugal. Se ha visto una relación estadísticamente significativa entre el consumo de esos fármacos y el aumento de resistencias. ${ }^{9}$ De la misma manera, esos estudios demuestran una baja resistencia a FT en toda Europa incluyendo el EC. La generación de resistencias como resultado del uso indiscriminado tiene como consecuencia que antibióticos clásicamente utilizados en la profilaxis y tratamiento de procesos urológicos tales como las Cefalosporinas de $2^{\text {a }}$ generación, TMP + SMX o incluso las Quinolonas, no puedan ser recomendados actualmente para el tratamiento de primera línea de las ITU no complicadas. ${ }^{1,15,16}$
En el análisis de nuestra microflora mediante urinocultivos, coincidimos con las guías y la mayoría de estudios en que el EC es el patógeno más frecuente, pero hay un porcentaje de más del 13\% de Enterococci. Esos últimos presentan una resistencia natural Sulfamidas y Cefalosporinas. Además EC presentó unas resistencias a TMP-SMX y Ciprofloxacino de aproximadamente 30\%. Es por ello que en nuestro servicio administrábamos Gentamicina $240 \mathrm{mg}$ intramuscular como profilaxis de las cistoscopias basándonos en estudios anteriores. ${ }^{10}$

Un antibiótico de uso profiláctico ha de reunir una serie de características: vida media de eliminación prolongada, poca transformación hepática, elevada eliminación por vía renal, mínima resistencia cruzada, no activa frente a patógenos especiales, baja toxicidad y bajo coste. ${ }^{17}$

La Fosfomicina es un derivado del ácido fosfónico. Es excretada por la orina principalmente mediante filtración glomerular. Tras la administración de una única dosis de FT $3 \mathrm{~g}$ los picos de concentración plasmática se alcanzan en unas 2 horas, y el principal pico de concentración urinaria se recupera a las 4 horas de su administración. Esas cifras no se modifican con la insuficiencia renal leve o moderada ${ }^{18}$ En algunos estudios se han intentando administrar doble dosis, una antes y otra 24 horas después del procedimiento, pero no ha demostrado ser más eficaz y sí han aumentado los efectos adversos. ${ }^{19}$

El 90\% de las cepas de los uropatógenos más frecuentes con el EC, Klebsiella spp, etc, son sensibles a la Fosfomicina. Y los estudios epidemiológicos europeos no han observado un aumento de resistencias a FT en pacientes con ITU no complicadas en los últimos 8 años permaneciendo por debajo del $3 \% .{ }^{4}$ Además, la resistencia cruzada con Fluoroquinolonas es bastante rara y permanece activa en presencia de Enterobacteriaceae productoras de $\beta$-lactamasas. ${ }^{20}$

Los efectos adversos no suelen ser frecuentes y si los hay no suelen ser graves. Los más frecuentes que recoge la literatura son: la diarrea (4\%) y el dolor de cabeza (2\%), que aumentan su incidencia con el incremento de la dosis. ${ }^{20}$ En nuestro estudio, ningún paciente refirió efectos secundarios tras la toma de la medicación.

Tradicionalmente se considera que el periodo ideal para la administración de la profilaxis es 1 o 2 horas antes del procedimiento, pero existe bibliografía que defiende que se obtiene la misma eficacia si se administra hasta 3 horas después del procedimiento. ${ }^{21,22}$ La vía oral está recomendada en aquellos procedimientos menores a los que se les pueda dar el antibiótico 1 hora antes de la prueba. ${ }^{5}$ En nuestro estudio, los pacientes se tomaban la FT $3 \mathrm{~g}$ vía oral entre 15 y 20 minutos antes de la prueba mientras estaban en nuestra sala de espera con el objetivo de asegurar el cumplimiento por parte del paciente.

La mayoría de los estudios sobre quimioprofilaxis utilizan las Fluoroquinolonas o el TMP-SMX, y en menor medida la Cefalosporinas como antibiótico de elección. ${ }^{6,23}$ Existen pocos estudios en los que utilicen la FT $3 \mathrm{~g}$ como profilaxis antes de la cistoscopia. Los más destacados de Nicoletti y col. ${ }^{19}$ y el de Jiménez-Pacheco y col., ${ }^{12}$ presentan una muestra inferior y solo Periti y col. ${ }^{24}$ con 283 pacientes se aproxima a la nuestra, pero el suyo no era un estudio 
randomizado e incluía profilaxis en pacientes que iban a ser sometidos a resección transuretral.

Uno de los debates que suscitan siempre esos estudios, es determinar si las bacteriurias significativas postcistoscopias se consideran ITU que pueden requerir un tratamiento antibiótico, o si son meras bacteriurias asintomáticas las cuales no precisan de más actuación. ${ }^{6,23}$ Para poder diferenciarlas, en nuestro estudio se les avisó telefónicamente a los pacientes para saber si tuvieron síntomas urinarios tras la realización de la cistoscopia flexible, duración de los mismos y si fue necesaria actuación médica. Y se definió como ITU la presencia de bacteriuria significativa junto con la presencia de síntomas urinarios.

La literatura recoge una tasa de bacteriurias previas a la cistoscopia de cerca del $4 \%{ }^{25}$ que posteriormente se pueden perpetuar tras la realización de la prueba y pueden verse representados en el urinocultivo realizado 10 días después alterando los resultados. Sin embargo, hay ensayos en los cuales no se determina con un cultivo previo a la cistoscopia flexible la presencia de bacteriuria significativa o de síntomas del tracto urinario. ${ }^{23}$ En nuestro estudio, para poder determinar las verdaderas nuevas ITU tras la realización de la cistoscopia, se les realizó un urinocultivo previo a la cistoscopia y se les hizo una encuesta sobre la presencia de síntomas urinarios, y no se contabilizaron aquellos pacientes con criterios de ITU postcistoscopia que presentaban criterios de ITU previos a la cistoscopia.

En conclusión, la quimioprofilaxis previa a la cistoscopia con Fosfomicina Trometamol $3 \mathrm{~g}$ vía oral administrada a los pacientes con factores de riesgo de adquirir infecciones urinarias, es tan efectiva como la quimioprofilaxis con un antibiótico de amplio espectro de eficacia contrastada como la Gentamicina $240 \mathrm{mg}$ intramuscular. La FT es de fácil administración, tiene pocos efectos secundarios y es una alternativa válida para entornos con una flora que presente alta resistencia a antibióticos. No obstante, es recomendable ajustar el uso de antibióticos para la quimoprofilaxis según el estudio previo de los patógenos más frecuentes de cada entorno.

Responsabilidades Éticas

Protección de personas y animales. Los autores declaran que para esta investigación no se han realizado experimentos en seres humanos ni en animales.

Confidencialidad de los datos. Los autores declaran que han seguido los protocolos de su centro de trabajo sobre la publicación de datos de pacientes.

Derecho a la privacidad y consentimiento informado. Los autores declaran que en este artículo no aparecen datos de pacientes.

\section{Nota}

Los autores firmantes declaramos que se han obtenido los datos siguiendo los protocolos establecidos en nuestro centro sanitario. De la misma manera, declaramos no tener ningún conflicto de intereses, que no se ha reproducido total o parcialmente el contenido de este artículo y que no se ha duplicado o publicado en otras revistas.

\section{Referencias}

1 European Association of Urology (EAU). Guidelines on urological infections. Arnhem: EAU; 2015. Disponible en URL: https:// uroweb.org

2 Almallah YZ, Rennie CD, Stone J, Lancashire MJ. Urinary tract infection and patient satisfaction after flexible cystoscopy and urodynamic evaluation. Urology 2000;56(01):37-39

3 Burke DM, Shackley DC, O'Reilly PH. The community-based morbidity of flexible cystoscopy. BJU Int 2002;89(04):347-349

4 Cundiff GW, McLennan MT, Bent AE. Randomized trial of antibiotic prophylaxis for combined urodynamics and cystourethroscopy. Obstet Gynecol 1999;93(5 Pt 1):749-752

5 Mirone V, Franco M. Clinical aspects of antimicrobial prophylaxis for invasive urological procedures. J Chemother 2014;26(Suppl 1): S1-S13

6 Garcia-Perdomo HA, Jimenez-Mejias E, Lopez-Ramos H. Efficacy of antibiotic prophylaxis in cystoscopy to prevent urinary tract infection: a systematic review and meta-analysis. Int Braz J Urol 2015;41(03):412-424, discussion 424

7 Wagenlehner FM, Wagenlehner C, Schinzel S, Naber KG; Working Group "Urological Infections" of German Society of Urology. Prospective, randomized, multicentric, open, comparative study on the efficacy of a prophylactic single dose of $500 \mathrm{mg}$ levofloxacin versus $1920 \mathrm{mg}$ trimethoprim/sulfamethoxazole versus a control group in patients undergoing TUR of the prostate. Eur Urol 2005;47(04):549-556

8 Andreu A, Alós JI, Gobernado M, Marco F, de la Rosa M, GarcíaRodríguez JA; Grupo Cooperativo Español para el Estudio de la Sensibilidad Antimicrobiana de los Patógenos Urinarios. Etiología y sensibilidad a los antimicrobianos de los uropatógenos causantes de la infección urinaria baja adquirida en la comunidad. Estudio nacional multicéntrico. Enferm Infecc Microbiol Clin 2005;23 (01):4-9

9 Kahlmeter G. Prevalence and antimicrobial susceptibility of pathogens in uncomplicated cystitis in Europe. The ECO.SENS study. Int J Antimicrob Agents 2003;22(Suppl 2):49-52

10 Rané A, Cahill D, Saleemi A, Montgomery B, Palfrey E. The issue of prophylactic antibiotics prior to flexible cystoscopy. Eur Urol 2001;39(02):212-214

11 Olson ES, Cookson BD. Do antimicrobials have a role in preventing septicaemia following instrumentation of the urinary tract? J Hosp Infect 2000;45(02):85-97

12 Jiménez-Pacheco A, Lardelli Claret P, López Luque A, Lahoz-García C, Arrabal Polo MA, Nogueras Ocaña M. Randomized clinical trial on antimicrobial prophylaxis for flexible urethrocystoscopy. Arch Esp Urol 2012;65(05):542-549

13 Winstanley TG, Limb DI, Eggington R, Hancock F. A 10 year survey of the antimicrobial susceptibility of urinary tract isolates in the UK: the Microbe Base project. J Antimicrob Chemother 1997;40 (04):591-594

14 Trienekens T, Stobberingh E, Beckers F, Knottnerus A. The antibiotic susceptibility patterns of uropathogens isolated from general practice patients in southern Netherlands. J Antimicrob Chemother 1994;33(05):1064-1066

15 Aguiar JM, Chacon J, Canton R, Baquero F. The emergence of highly fluoroquinolone-resistant Escherichia coli in communityacquired urinary tract infections. J Antimicrob Chemother 1992;29(03):349-350

16 Garau J, Xercavins M, Rodríguez-Carballeira M, y col. Emergence and dissemination of quinolone-resistant Escherichia coli in the community. Antimicrob Agents Chemother 1999;43(11): 2736-2741

17 Dalet F, Del Rio G. Profilaxis antibiótica en Urología. Infecciones Urinarias, Barcelona; 1997:445-475

18 Wagenlehner FM, Thomas PM, Naber KG. Fosfomycin trometamol $(3,000 \mathrm{mg})$ in perioperative antibiotic prophylaxis of healthcareassociated infections after endourological interventions: a narrative review. Urol Int 2014;92(02):125-130 
19 Nicoletti G, Nicolosi D, Schito GC, Varaldo M, Carati L. Fosfomycin trometamol in prophylaxis of bacteriuria associated with transurethral diagnostic procedures. Urogynaecologia 1994;8:123-134

20 Fosfomycin Trometamol. Product Monograph. Milan, Springer Healthcare Italia, 2012

21 Classen DC, Evans RS, Pestotnik SL, Horn SD, Menlove RL, Burke JP. The timing of prophylactic administration of antibiotics and the risk of surgical-wound infection. N Engl J Med 1992;326(05): 281-286

22 Bates T, Siller G, Crathern BC, et al. Timing of prophylactic antibiotics in abdominal surgery: trial of a pre-operative versus an intra-operative first dose. Br J Surg 1989;76(01):52-56
23 Carey MM, Zreik A, Fenn NJ, Chlosta PL, Aboumarzouk OM. Should We Use Antibiotic Prophylaxis for Flexible Cystoscopy? A Systematic Review and Meta-Analysis. Urol Int 2015;95(03):249-259

24 Periti P, Novelli A, Reali EF, Del Bono GP, Fontana P. Prophylactic chemotherapy with fosfomycin trometamol salt in transurethral prostatectomy. A controlled clinical trial; in Neu HC, Williams JD (eds): New Trends in Urinary Tract Infections. The Single-Dose Therapy International Symposium, Rome, January 1987. Basel, Karger, 1988, pp 207-233

25 Johnson MI, Merrilees D, Robson WA, et al. Oral ciprofloxacin or trimethoprim reduces bacteriuria after flexible cystoscopy. BJU Int 2007;100(04):826-829 